\title{
Identifying and Structuring Skeletal Noise
}

\author{
Thomas Delamé, Céline Roudet, and Dominique Faudot \\ Le2i - Burgundy University, Dijon, France \\ tdelame@gmail.com \\ celine.roudet@u-bourgogne.fr \\ dominique.faudot@u-bourgogne.fr
}

\begin{abstract}
A skeleton is a thin centered structure within an object, which describes its topology and its geometry. The medial surface is one of the most known and used skeleton formulation. As other formulations, it contains noise, which complexifies its structure with useless parts. The connectivity of a skeleton is then unpredictable due to these useless parts. It can be a problem to segment the skeleton into logical components for example. We present here a technique whose purpose is to identify and structure such skeletal noise. It only requires a skeleton as input, making this work independent from any skeletonization process used to obtain the skeleton. We show in this paper that we significantly reduce the skeletal noise and produce clean skeletons that still capture every aspects of a shape. Those clean skeletons have the same local topology as the input ones, but with a clearer connectivity.
\end{abstract}

Keywords: skeleton, medial surface, skeletal noise, hairy pattern

\section{Introduction}

A skeleton is a thin structure centered within an object, describing the topology and the geometry of this object. Such a skeleton could be then used as a shape representation model for every closed object. There exist several types of skeletons and we could divide them into two main categories:

1. curve skeletons; they are composed of curves and used for shape registration [5], mesh segmentation [4] and data reconstruction [18].

2. surface skeletons; they are composed of curves and surfaces, among them we can find medial surfaces [6], Midpoint loci [8] and PISA axes [16].

The work presented here is part of a process to make skeletons useful shape representation models. As stated in [11], surface skeletons better capture the geometry of objects than curve ones, making them best candidates for this purpose. Thus, we consider only surface skeletons, and in particular medial surfaces, because they are well defined and many algorithms exist to efficiently approximate them. A medial surface is made of atoms. An atom is a maximal inscribed ball lying inside the described object. Each atom $a$ is connected to other atoms, called its neighbors $\mathcal{N}(a)$. Those links confer a topology on the skeleton. 
Skeletons are obtained from objects through a process called skeletonization. In such a process, a finite number of samples is used to capture an object $\mathcal{O}$. These samples cannot capture completely $\mathcal{O}$, and uncertainty about the object boundary arises: do a set of close samples capture a feature or a smooth part? In each case, atoms will be inserted in the skeleton. Atoms that do not capture any features produce the skeletal noise. Many methods have been proposed to remove unwanted components associated with this skeletal noise like [13] or [1]. A key goal of such methods is to preserve the topology of the skeleton during the process.

We noticed that the skeletal noise can be classified into two categories: Type 0 noise, also called clusters, and Type 1 noise, commonly referred to as hairy pattern. These noise types make the use of skeletons uneasy as shape representation models. For example, it is hard to estimate the tangent plane to an atom if one of its neighbors $a$ belongs to Type 1 noise, since atoms such as $a$ do not lie near the medial surface. Also connections are unnecessarily complex due to these noises. Connections get even worse when we look closely inside a cluster, locus of Type 0 noise, where the union of all its balls could be perfectly described by only one ball. The Figure 1 illustrates this classification.

In this paper, we propose new criteria to identify those skeletal noise. We also present a skeleton structure that contains a hierarchy, used to reflect the importance of atoms. Atoms are labeled by three number: 0 for Type 0 noise, 1 for Type 1 noise, and 2 otherwise. The hierarchy isolates skeletal noise from the remaining skeleton atoms: atoms from skeletal noise cannot have connections with relevant atoms. The neighbors are reorganized due to this isolation, in order to preserve the skeleton structure (i.e. logical components of the skeleton) thanks to structuring rules we created. There is no requirement about how the input skeleton was obtained, as our method take place apart from any skeletonization process. Thus, our work is general enough to be applied to every connected skeleton. More importantly, we show that our cleaned skeleton is simpler with a clearer connectivity, while not loosing any features or the original skeleton.

\section{Previous Work}

Since medial surfaces had always been noisy, removing this noise from it had motivated a lot of work. This is generally done during the skeletonization process: when the skeletonized object $\mathcal{O}$ is known. Thus, a noise removal technique is strongly linked to a skeletonization method. The most popular approaches for skeletonization are based on the Voronoi diagram or its dual Delaunay tetrahedralization. We review here such noise removal techniques.

Because such techniques are related to skeletonization, they must ensure that they provide a good skeleton. A good skeleton may be seen as a skeleton which converges to the medial surface when the sampling density of $\mathcal{O}$ tends to infinity. A very important result in $3 \mathrm{D}$ is that, unlike in $2 \mathrm{D}$ [9], the Voronoi vertices do not converge to the medial surface, as the sampling density tends to infinity [2]. 


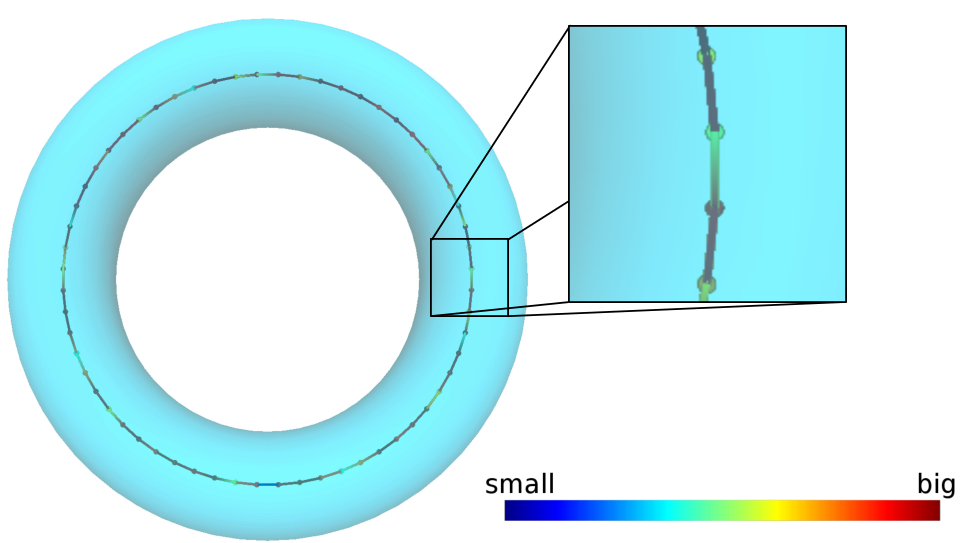

a)

b)

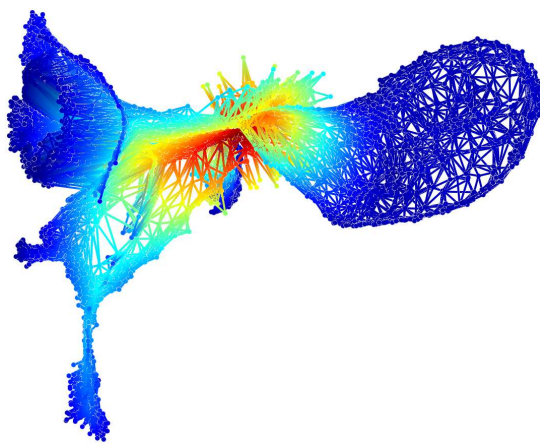

c)

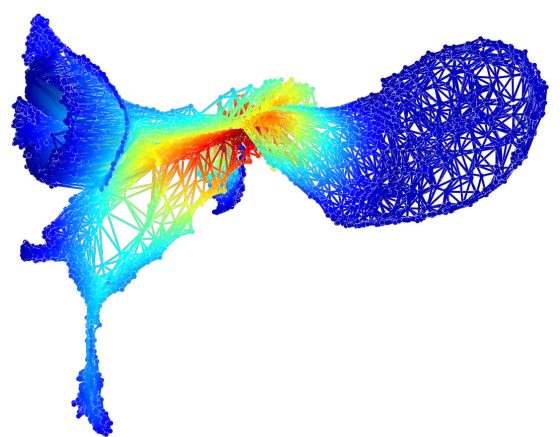

d)

Fig. 1. Illustration of skeletal noise. a) This torus has $94 \%$ of its atoms belonging to Type 0 noise, such that the englarged view contains 68 atoms, while only 4 can be seen. b) To represent skeletons in this paper, we use a color code to transcript the radius of an atom: from blue for small values to hot colors for big values. c) The blender monkey model's skeleton contains hairy pattern, especially at the center of the skull. d) The cleaned skeleton of the monkey model obtained with our method.

An approach that guarantees convergence uses a subset of the Voronoi vertices, named the poles [1]. For a sample point $p$, the poles are the vertices of its Voronoi polyhedron that are the furthest away from $p$ on the two sides of the surface. The balls centered in these poles (with radii equal to the distance to their samples) are called polar balls. The method is very robust and the produced skeleton, called the Power Shape, is visually reasonable. However, skeletal noise remains and many flat tetrahedra populate the skeleton. Also, this method was firstly intended to reconstruct a shape from a point cloud. Thus, it does not take advantage of any information contained in $\mathcal{O}$ except some sample positions.

Dey and Zhao [13] presented a method where (a subsequent part of) the output converge to the medial surface, by applying angle-based filter conditions to the Voronoi diagram. The filter parameters are scale and density independent. However the skeleton topology is ignored and some holes appear in many cases, inducing loss of information or topology changes in the described object. 
In [10], the authors introduce the notion of weak feature size $\mathcal{F}(x)$ at a point $x$. This is the radius of the minimal ball enclosing closest object boundary points to $x$. If we remove the Voronoi vertices $v$ with $\mathcal{F}(x)<\lambda$, we obtain the $\lambda$-Medial Axis. The main disadvantage with such technique is the definition of $\lambda$ for a shape: as $\lambda$ increase, more skeletal noise is removed, as well as some features.

Another method called the Scale Axis [17] produces very nice looking skeletons. This work is based on the Power Shape, with useful enhancements. The input object is remeshed and sampled by the technique proposed in [7]. Polar balls are efficiently obtained from an input mesh thanks to a more adapted process. Those polar balls are scaled by a factor $s$. The skeleton, composed of the union of those scaled balls, is computed and then cleaned by a topology-preserving angle filtering. Finally, the balls in the skeleton are scaled back by a factor $1 / s$. The scaling factor allows the skeletal noise removal by a spatially adaptive feature classification. With some constraints on the $s$ parameter, the Scale Axis transform has been proven to have topological stability guarantees [14].

We propose in this paper to perform the noise removal apart from the skeletonization, working directly on the skeleton with no further information. As shown in the result section, our technique significantly remove the skeletal noise and keeps every details. Moreover, it maintains the topology of the skeleton.

\section{Outline}

In this paper, we first describe the model we have conceived to structure the skeleton (Section 3). We also present the atomic operations that can be realized on this model (Section 3.3). Then, we detail the methodology we used to identify skeletal noise of Type 0 (Section 4) and Type 1 (Section 5). We finally expose our validation process, and conclude.

\section{Structuring Model}

In this section, we present the skeleton model used to identify and structure the skeletal noise.

\subsection{Atoms}

Basically, atoms can be seen as balls whose union approximates an object. We consider them as nodes of a graph: they have a position, a radius, and links with other atoms. We add to this graph a hierarchy structure, reflecting the importance of the atoms. Each atom has then a rank that is equal to its level in the hierarchy: 0 for a useless atom, 1 for a unimportant atom, 2 for a more important and so on. The rank is the label of the atom node. Since we deal with a hierarchy, atoms may have also a father and sons. If an atom does not have a father, we call it root. Otherwise, we call it sub-atom. The Figure 2 shows a schematization of these concepts. 


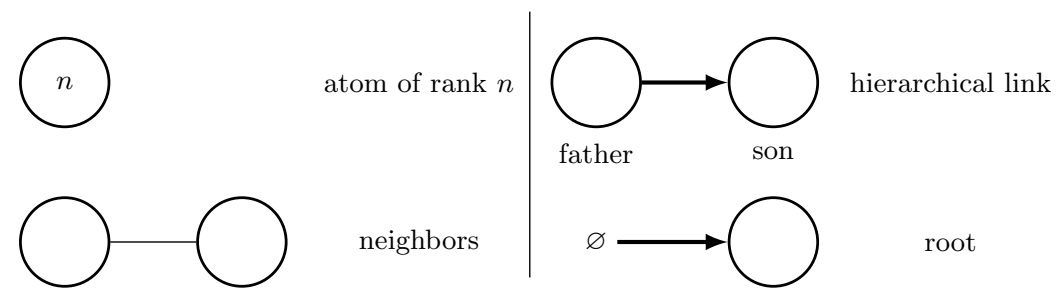

\subsection{Skeleton}

Fig. 2. Schematization of atoms

The skeleton has thus two structures: one composed of neighbor links (representing the topology), and another one composed of hierarchical links (reflecting the importance of atoms). In order to combine those two structures, we introduce the notion of $n$-hierarchy. A skeleton is said to be a $n$-hierarchy if:

i) an atom is a root $\Longleftrightarrow$ an atom has rank $n$,

ii) two atoms have different ranks $\Longrightarrow$ they cannot be neighbors,

iii) every sub-atom has a rank lower than the one of its father.

The structuring process starts with a 0-hierarchy skeleton $\mathcal{S}$ : a graph where each node is 0 labeled. Then $\mathcal{S}$ is structured rank by rank, using criteria to detect when an atom $a$ is less important than another atom $b$. The rank of such atom $b$ is changed to reflect its importance relatively to $a$. Such an operation make impossible to keep the $n$-hierarchy property because there will be be roots at ranks $n$ and $n+1$ (See Figure 3). We hence release some of the constraints contained in the definition, to introduce the notion of $n$-consistent skeleton. This intermediary state specify how the skeleton should be before reaching a hierarchy of higher level. Such a skeleton meet the following requirements:

i) roots can only be at the levels $n$ and $n+1$, and every atom of rank $n+1$ is a root,

ii) only two roots or two atoms with the same rank can be linked,

iii) every sub-atom has a lower rank than its father.

The roots of level $n+1$ are called the processed roots, because they will remain roots of level $n+1$ until the skeleton becomes a $n+1$-hierarchy. Because of $i$ ), atoms corresponding to noise are not linked to important atoms. It is a way to isolate them. Items i) and iii) make the roots of a skeleton the most important atoms. If we consider only these roots we have a connected skeleton called in this paper clean skeleton, since the low ranks contain skeletal noise.

\subsection{Structuring Operations}

When an atom $a$ is detected as less important than another atom $b$, we perform a structuring operation on the skeleton, called absorption and written $b \succ a$, to reflect this information. For each kind of addressed importance, a specific absorption is defined. In this paper, we deal only with importance relatively to noise. Thus we define one absorption for clusters and one for hairy pattern. 


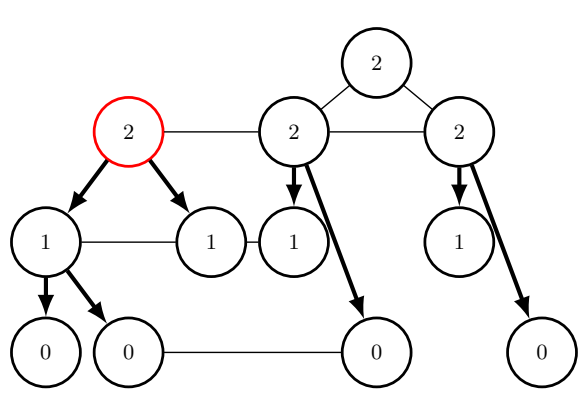

a)

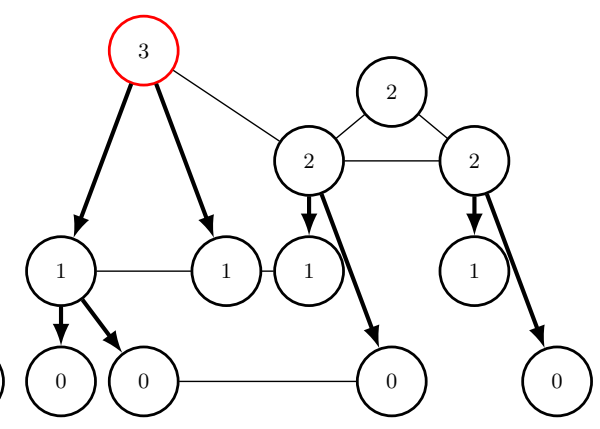

b)

Fig. 3. Schematizations of skeletons. a) This skeleton is a 2-hierarchy. The atom in red is promoted to a higher rank because we detected it as important. We obtain the situation b), where this skeleton is 3 -consistent.

An absorption always sets the less important atom $a$ as the son of a root atom $c$.primitive $c \rightarrow a$. After such action, $c$ must update its rank $r_{c}$ to have the same rank as the processed roots, i.e. $n+1$. The rank $r_{c}$ is then greater than the rank of $a$, in order to meet the constraint described in iii). Then, every link between a sub-atom and $c$ must be removed, to fulfill item ii). The last two operations are called promotion and written promote $(c)$.

In order to maintain the skeleton topology, during the absorption, the links of $a$ are transmitted to $c$. Moreover, we also need to remove the links between $a$ and the other roots in the hierarchy (item ii)).

\section{Identifying and Structuring Atom Clusters}

This section deals with atom clusters and proposes a solution to identify them and structure them.

\subsection{Observations About Atom Clusters}

We know exactly the theoretical skeletons of simple objects, e.g. torus, sphere. When we observe the practical skeletons obtained for such objects, we can notice a huge difference between the number of perceived atoms and the real number of atoms. This difference is due to the presence of clusters: a high number of atom loci is contained in a very small spatial area. In such places, we perceive only one atom (at a reasonable scale), while in practice, there are so many, as shown in Figure 1 a).

Atoms are maximal inscribed balls. Thus no atom can be fully contained into other ones. If we suppose there is a difference of radii within a cluster, the biggest atom would contain at least one atom, as they are very close. This is a contradiction, and then every atom in a cluster has a similar radius. So, every atom in the cluster adds a very tiny piece of information (about the size of the machine precision). Consequently, a cluster should then be replaced by one of 
its atoms since it is useless to store a large number of atoms that add almost nothing.

Clusters are due to parts that are locally spherical. Basically, with a Voronoi diagram based skeletonization technique, each 4 cospherical samples produce an atom, located at the circumsphere. If there are more than 4 cospherical samples, duplicated atoms are created. Due to machine precision, duplicated atoms will not be at the same location, but very close and with very similar radii. Clusters could be then addressed in the skeletonization process by identifying samples that are cospherical, like in [19]. In the next section, we will show an identification criterion that is independent from the skeletonization technique.

\subsection{Identification Criterion}

Since atoms inside the same cluster are very close and have almost the same radius, the volume $\mathcal{V}_{a \backslash b}$ added by an atom $a$ to another atom $b$ of the same cluster is nothing compared to the volume $\mathcal{V}_{b}$. We define a test $\mathcal{F}_{0}(a, b)$ which indicates whether $a$ and $b$ belong to the same cluster and if $a$ is less important than $b$. Here is the expression of the test $\mathcal{F}_{0}$ :

$$
\mathcal{F}_{0}(a, b)=(a \cap b \neq \emptyset) \bigwedge\left(\mathcal{V}_{a}<\mathcal{V}_{b}\right) \bigwedge\left(\mathcal{V}_{a \backslash b}<\kappa \cdot \mathcal{V}_{b}\right)
$$

This test allows to detect clusters with only one parameter, $\kappa$, which is easy to understand. Using a relative measure based on the volume of an atom makes this criterion insensitive to scale while being local. Thus, it suits models of any size while taking into account the local thickness of the shape, to avoid the labeling of small details as Type 0 noise. In our implementation, we noticed that $\kappa=5 \%$ o is enough for all the tested objects.

There is no requirement on links between $a$ and $b$, and we explain here why. First, there is no need to look at links to identify a cluster. Second, links inside a cluster are chaotic, we cannot rely on them for efficiency purpose. Finally, there exist isolated vertices inside a cluster for skeleton based on the Power Shape algorithm (due to the use of a regular tetrahedralization to define the links). Ignoring links when detecting clusters allows us to remove isolated vertices from the cleaned version of a skeleton.

\subsection{Structuring Process}

We process every atom of rank 0 such that there is no cluster in the clean skeleton, i.e. among the roots. For a cluster, only one of atom $a$ will reach the rank 1, while every other atom will be the sons of $a$. Atoms that do not belong to a cluster are promoted to the next rank at the end of the process such that we obtain a 1-hierarchy.

To process an atom $a$, we use the test function $\mathcal{F}_{0}$ with already processed atoms $\mathcal{P}$. For $b \in \mathcal{P}$, if $\mathcal{F}_{0}(a, b)=$ true, then $a \prec b$, otherwise if $\mathcal{F}_{0}(b, a)=$ true, then $b \prec a$. 
For clusters, the absorption is context dependent: the result is not the same depending whether input atoms are roots or not. We give a summary of structuring rules for this absorption in the Figure 4 . For reasonable values of $\kappa$, this process keeps the skeleton topology because only very close atoms are removed (and links with removed atoms are transmitted to their fathers). Also, there is no need to impose a processing order, as every atom in a cluster is interchangeable.

\section{$5 \quad$ Identifying and Structuring Hairy Pattern}

This section deals with hairy pattern and propose a solution to identify them and structure them.

\subsection{Observations About Hairy Pattern}

The hairy pattern is one of the most known and recognizable skeletal noise. It consists in atoms that do not capture any feature. They are produced by circumspheres that have some of their 4 spherical sample points close from each other. This is why the Lambda Axis [10] prune atoms when distances between these samples are below a threshold.

When we explore the skeleton looking for Type 1 atoms, we notice something about their neighbors: they are located in a narrow cone, and most of them belong to the stable skeleton. The stable skeleton is the visually free of noise skeleton. This neighbor configuration gives to Type 1 atoms a spiky appearance, making them off-centered. Also, such atoms have a radius lower than their neighbors on the stable skeleton. So when we move from a Type 1 atom toward the stable skeleton, the radii of atoms get bigger.

The ends of thin skeleton parts, e.g. for fingers, are very similar to hairy pattern. The distinction is made with the length of such pattern: if this length is a small compared to surrounding parts, we have a hairy pattern. So, to deal with hairy pattern, we should limit the length of absorbed skeleton components, to avoid the classification of thin detail parts as noise.

\subsection{Identification Criterion}

Since Type 1 atoms are off-centered, we have built a simple criterion based on this. We check the location of the neighbors of an atom $a$ by computing a sphere $\mathcal{B S}(a)$, which is the minimal sphere containing their positions. If $a$ is not inside $\mathcal{B S}(a)$, we consider that $a$ is off-centered and thus is a Type 1 atom. This parameter-free criterion is better suited than setting a threshold to control the thickness of the cone containing the neighbors.

To define $\mathcal{B S}(a)$, the atom $a$ must have at least 2 neighbors. Otherwise, $a$ has one neighbor $b$ : it can either be noise or the termination of a thin curvilinear component. In the latter case, $a$ should not be absorbed. We distinguish those cases by counting the neighbors of $b$. If it is more than $2: b$ is not a part of a thin curvilinear component and $a$ is not absorbed. This gives us the following test $\mathcal{F}_{1}$ to identify a Type- 1 atom: 


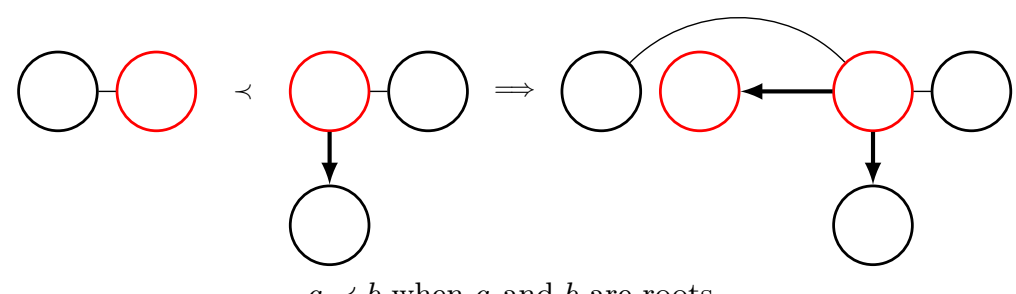

$a \prec b$ when $a$ and $b$ are roots.
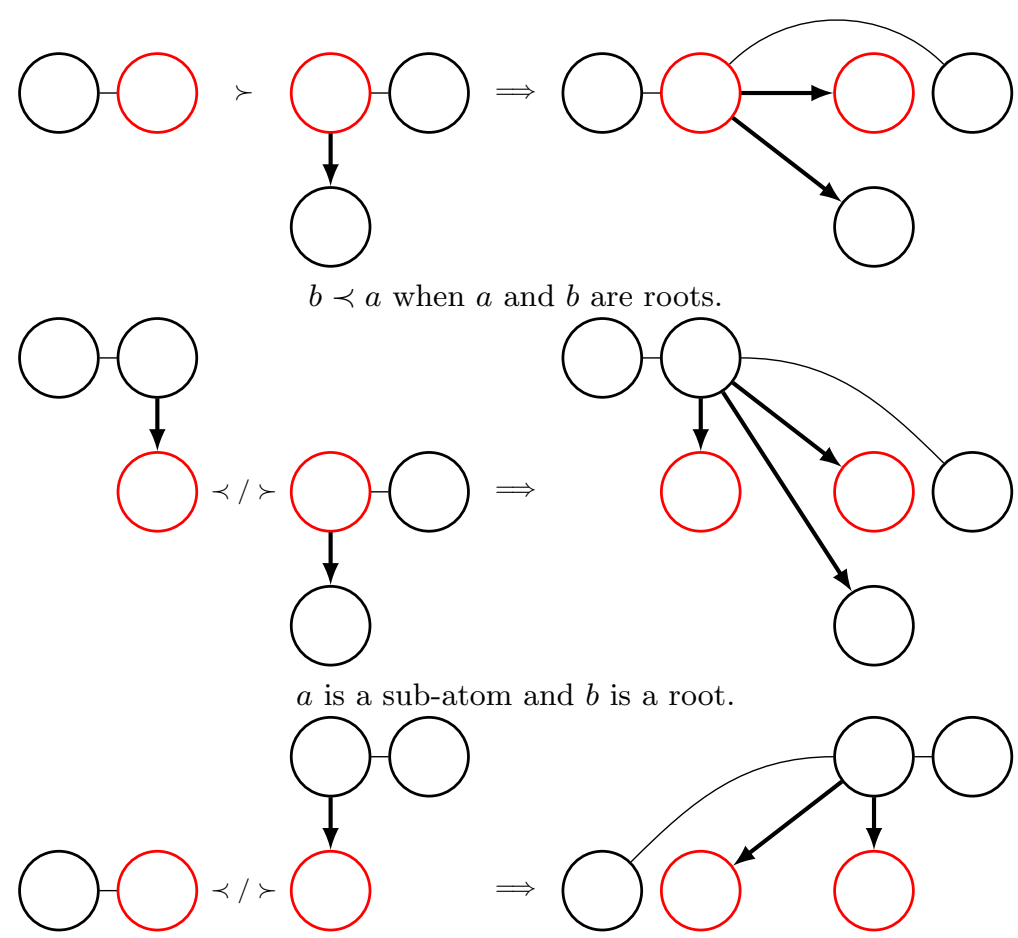

$a$ is root and $b$ is a sub-atom.

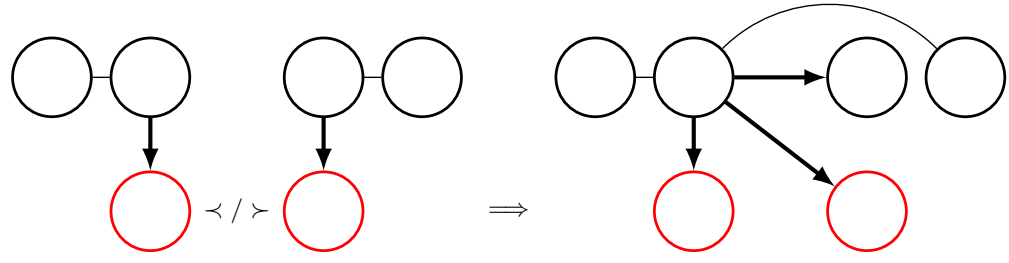

$a$ and $b$ are sub-atoms.

Fig. 4. Schematization of cluster absorptions. The atoms concerned by this operation are depicted in red, $a$ on the left that is the currently processed atom, and $b$ on the right which had already been processed. For simplicity purpose, we do not schematize the operations to meet the constraints described by item ii) in the definition of a $n$-consistent skeleton. 


$$
\mathcal{F}_{1}(a)=\left\{\begin{array}{c}
a \in \mathcal{B S}(a), \text { if } \sharp \mathcal{N}(a)>2 \\
\sharp \mathcal{N}(b)<3, \text { if } \mathcal{N}(a)=\{b\} \\
\text { false }, \text { in other cases }
\end{array}\right.
$$

\subsection{Structuring Process}

Initially, $\mathcal{S}$ is a 1-hierarchy. We have to test and structure the following set of atoms: $\mathcal{P}=\operatorname{Roots}(\mathcal{S})$. Every atom $a \in \mathcal{P}$ is re-tested when a change (an absorption) is made in $\mathcal{P}$. We assume that $\mathcal{F}_{1}(a)=$ true, otherwise we simply take the next atom in $\mathcal{P}$.

If $a$ has only one neighbor $b$ we realize the operation $a \prec b$. In the other case, we must choose among the neighbors of $a$, the atom $b$ to use in the absorption. We propose to set $b$ as the neighbor of rank 1 with the highest radius. This has two consequences:

- the absorption is made toward the stable skeleton because bigger atoms in a hairy pattern are closer to the stable skeleton than smaller atoms (see Section 5.1).

- choosing a rank 1 atom will limit the length of an absorbed part, since in combination with the identification criterion, there will be no further possible absorption. Thus thin detail skeleton parts will be protected, as shown in Figure 5 .

Structuring rules for the hairy pattern absorption are detailed in the the Figure 6. Due to the identification criterion, atoms at crossings of skeleton components or inside a component are not detected as hairy pattern: their neighbors are all around them, thus they are inside the minimal bounding sphere. Only atoms in the boundary of components and hairy pattern are detected. As there is an effect which limits the length of the absorption, no components can completely disappear. Moreover, the links of an absorbed atom are transmitted to its father, without any loss of connectivity information. So, the topological structure of the clean skeleton remains the same after this step.

We impose to process $\mathcal{P}$ from the lowest radius to the biggest one. The result is then the same no matter the creation of the skeleton structure.

\section{Validation}

We validated our work with quantitative and qualitative comparisons. These comparisons were made with two different skeletons for some input shapes. The results express the "compression" realized by our technique on skeletons, while quantifying the modification in the geometric data. Also, the qualitative study shows the improvement in the skeleton connections and the conservation of the skeleton structure. 


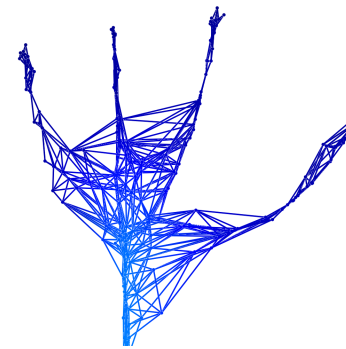

a)

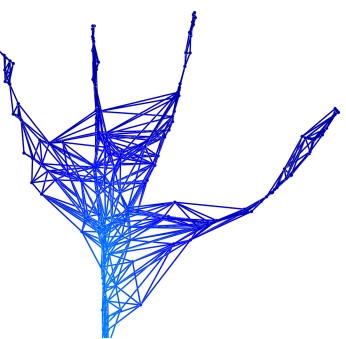

b)

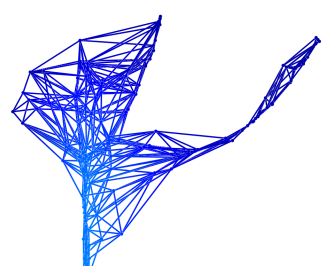

c)

Fig. 5. Effect of the hairy pattern structuring process. The input skeleton of Homer hand is presented in a). With the method described here we obtain the result b). If we allow any neighbor of rank 1 to absorb an atom detected as noise, fingers disappear as shown by c).

\subsection{Protocol}

We took some input shapes, and we skeletonized them by two well-known algorithms that give theoretical guarantees about the results while reducing the skeletal noise: the Power Shape and the Scale Axis. Each of these skeletons was structured by the technique we presented previously, giving us four skeletons by input shape.

We first computed statistics about those skeletons, to quantify their complexity. We also measured the distance between the input shape and the shape described by a skeleton. To do so, we used the Skin Surface [15], a garbing algorithm which only considers the spheres. This way, we measure the possible loss of geometric data in the cleaned skeleton relatively to the input skeleton. If we had used a garbing algorithm that consider other primitives to enhance the garbing mesh, like [12] that removes surface noise in the garbing mesh, the loss of geometric data would have been hidden. We chose the Root Mean Square distance (RMS), and we computed the distance between the input shape and the skin surface of each skeleton by the Mesh software [3].

Then, we compared visually the quality of the skeletons. We examined the links between atoms, looked for remaining noise and checked that detail was not removed.

\subsection{Quantitative Comparison}

First, Figure 7 expresses that the skeletons have been reduced with our structuring process. In average, only $85 \%$ atoms of the Power Shape remain in the clean skeleton, and $53 \%$ of the Scale Axis. For the Power Shape, the majority of the skeletal noise is caused by hairy pattern. However, such a skeletonization technique was firstly designed to remove such noise type. Thus, a noise removal algorithm done apart from the skeletonization, like ours, is useful to produce clearer skeletons, as noise still remains. 

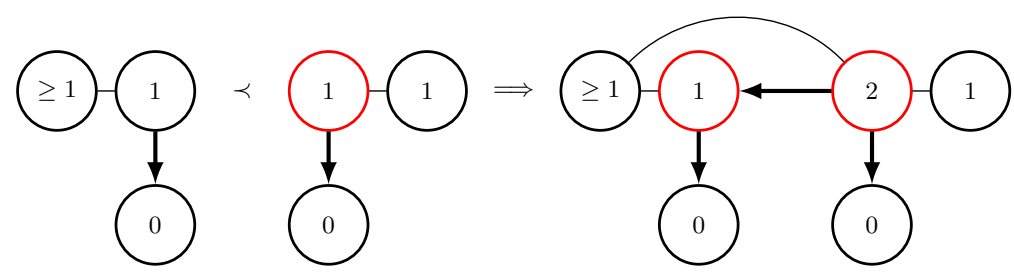

$a \prec b$ when $a$ and $b$ have rank 1 .

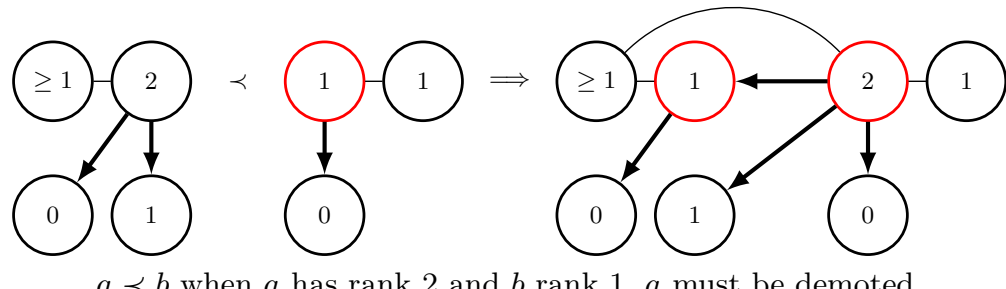

Fig. 6. Schematization of hairy pattern absorption. The atoms concerned by such operation are depicted in red, $a$ is on the left and $b$ on the right.

This statement is confirmed by the huge quantity of clusters contained in the Scale Axis. Such a skeleton is made of far more atoms than previous ones. A trend in skeletonization techniques is to sur-sample the input object, in order to produce more atoms. By doing so, we expect the skeleton to be more accurate and clear. As there are more atoms, they add fewer information to their neighbors, and our cluster identification criterion is triggered.

\begin{tabular}{|c|c|c|c|c|}
\hline & $\begin{array}{c}\text { Power } \\
\text { Normal }\end{array}$ & $\begin{array}{l}\text { Shape } \\
\text { Cleaned }\end{array}$ & $\begin{array}{r}\text { Scale- } \\
\text { Normal }\end{array}$ & $\begin{array}{l}\text {-Axis } \\
\text { Cleaned }\end{array}$ \\
\hline armadillo & 4.33 & 4.75 & 49.11 & 47.42 \\
\hline baby & 7.15 & 7.23 & 41.17 & 40.35 \\
\hline bimba & 7.36 & 7.41 & 82.24 & 80.94 \\
\hline boy & 11.20 & 11.31 & 39.85 & 38.75 \\
\hline bunny & 23.99 & 20.14 & 91.56 & 90.49 \\
\hline camel & 16.12 & 15.67 & 64.73 & 63.78 \\
\hline dinopet & 13.74 & 13.64 & 49.58 & 47.86 \\
\hline egea & 4.87 & 5.79 & 134.65 & 133.23 \\
\hline fish & 32.90 & 31.81 & 35.34 & 35.33 \\
\hline homer & 10.16 & 10.43 & 65.71 & 63.47 \\
\hline horse & 11.76 & 10.68 & 59.67 & 58.73 \\
\hline
\end{tabular}

Table 1. RMS distances between original object and skin surfaces of both normal and cleaned skeletons. Results are given in percent relatively to the diagonal of the bounding box.

One could argue than such results are obtained because we remove details from the skeletons. However, Table 1 prove that we only removed skeletal noise from the skeletons. The geometric data loss with our clean skeleton obtained for 

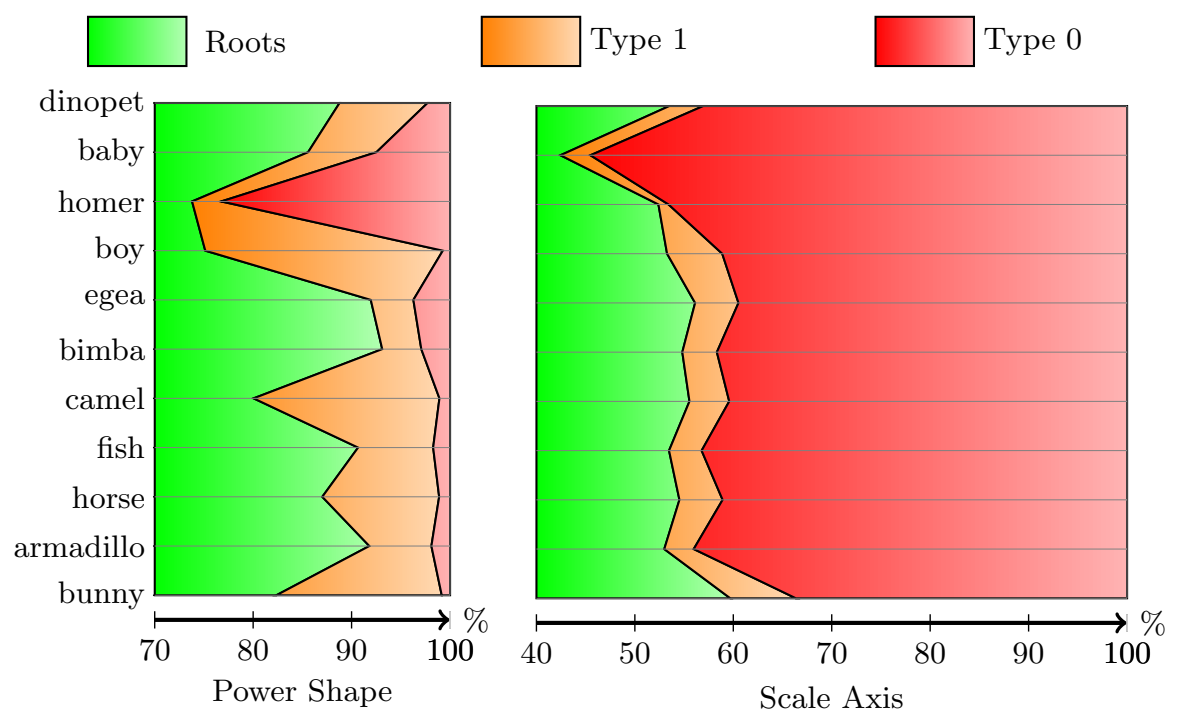

Fig. 7. Percentage of Type 0, Type 1 and roots in the cleaned Power Shape and the cleaned Scale Axis

the Power Shape is very small, about $1 \%$. For some cases this is even better, as skeletal noise can add material outside the original shape. For the Scale Axis, there is no loss of data reported: the RMS distance is always better. Thus our technique removed $47 \%$ of atoms in the Scale Axis without losing any detail compared to the input skeleton. This demonstrates that our technique efficiently remove skeletal noise from these skeletons.

The results also raised the question about the legitimacy of the actual trends in skeletonization: producing skeletons with more atoms does not improve the geometry captured, it mostly adds skeletal noise.

\subsection{Qualitative Comparison}

As clusters are by definition composed of atoms that cannot be distinguished visually, their absorptions make no visual difference. Also, we absorb the skeletal noise without any loss of detail, thus there is no missing parts in the skeleton. For these two reasons, there is not important visual differences between a skeleton and its cleaned version. We can only notice the removal of hairy pattern, erasing some small spiky component, and the disappearance of some atoms in skeleton boundaries.

For Scale Axis skeletons, the noise of Type 1 - the only visible noise - is closer to the stable skeleton. Thus, the visual enhancement on the clean skeleton is less than for the Power Shape. We checked the visual enhancement on a Power Shape obtained with much more samples to raise the number of atoms. As shown in Figure $8 \mathrm{~g}$ ) and $\mathrm{h}$ ), the skeleton is visually enhanced and the atom reduction 
is $53 \%$, with a relative proportion of Type 0 and Type 1 noise nearly the same as for the Scale Axis. So even if raising the number of atom in a skeleton increase the Type 0 noise, the Scale Axis reduces the visual importance of Type 1 noise, while our method reduces both noises in terms of quantity.

\subsection{Limitations}

There are two limitations to our technique. The first one is due to the constraint we have on detail. Indeed, to use skeletons as shape representation models in computer graphics applications, they should be able to capture small features. Thus, the cleaning process let untouched some hairy patterns to not absorb small features because small features can also be detected as hairy pattern. This effect can be handled by two or more ranks of hairy pattern when needed, but it requires the user intervention to determine when to stop.

The second limitation comes from the definition of the $\kappa$ value. If it remains small, the skeleton is perfectly clean and clear, no detail is loss. But with bigger values of $\kappa$, parts of the skeleton disappear, being iteratively absorbed. This is especially the case on models which have a lot of small features, like the Armadillo model, or on skeletons with highly dense atoms: an atom is very likely to add not enough material to its neighbors, and thus is absorbed. As the $\kappa$ value is meant to handle only clusters, i.e. machine precision issues, we highly discourage the use of high values of $\kappa$ : the skeleton will lose its structure and features will be missing.

\section{Conclusion \& Future Work}

In this paper, we addressed the removal of skeletal noise, i.e. atom clusters and hairy pattern. Such noise produces unnecessarily complex skeleton, by raising its data size and disturbing its structure. Unlike the numerous methods that take place in a skeletonization algorithm, our technique can be directly used on any connected skeleton, requiring no additional data like the original shape or angle values stored with atoms. Another originality of our work, is the use of a hierarchy structure to process the skeletal noise. Depending on its contribution to the geometric data of a skeleton, an atom receive a rank, reflecting its importance. Less important atoms are isolated from more important ones by this structure.

We showed in this paper how to take advantage of this hierarchy to clean a skeleton, in order to use it as a true shape representation model and not only an intermediary processing model. Indeed, atom clusters are removed, and the majority of hairy pattern is erased, while the skeleton do not suffer from any loss of detail. Even on input skeletons produced by skeletonization algorithms that are known to remove skeletal noise, we significantly reduce the number of atoms. The components of the skeletons remain the same, and no "hole" appears.

We define a level of detail, by considering only atoms with a rank greater than a minimal one. Once we get rid of the skeletal noise, we can identify and structure the features of the skeleton the same way we dealt with noise. This 


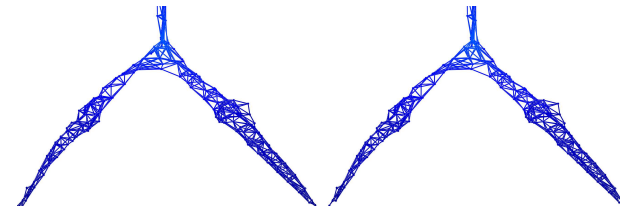

a)

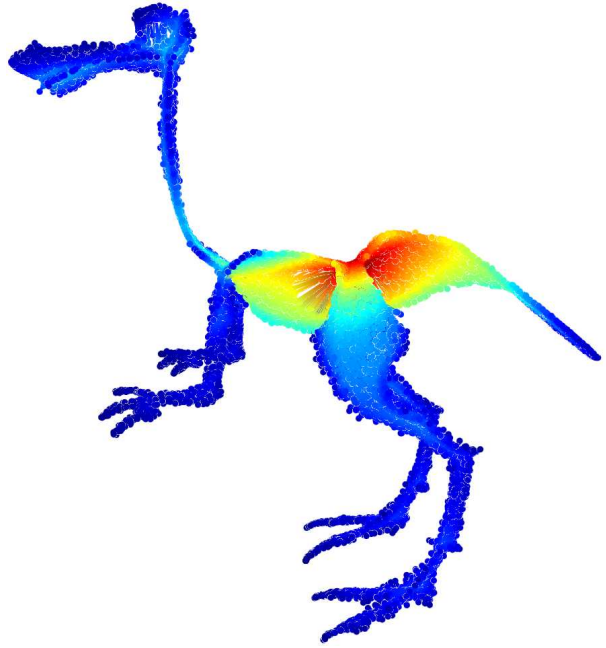

e)

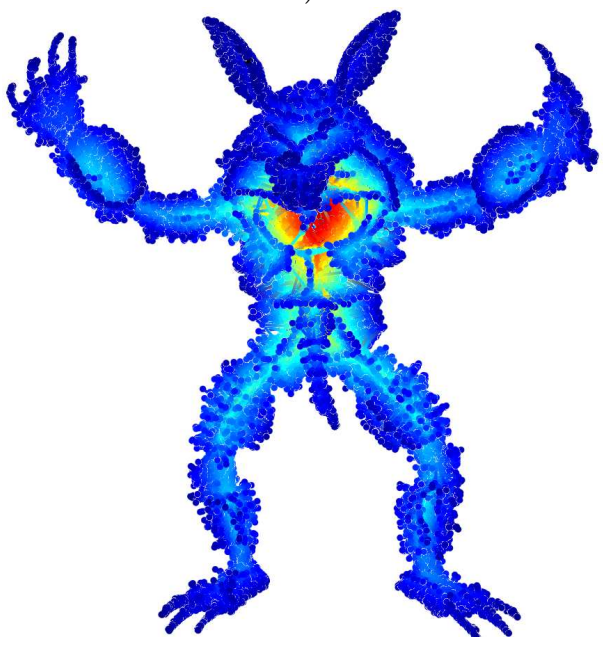

g)

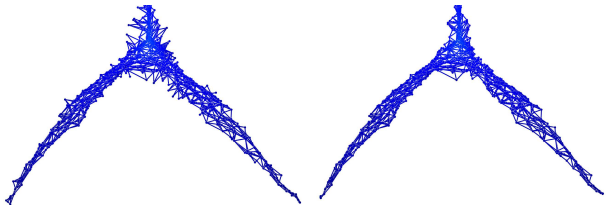

c)

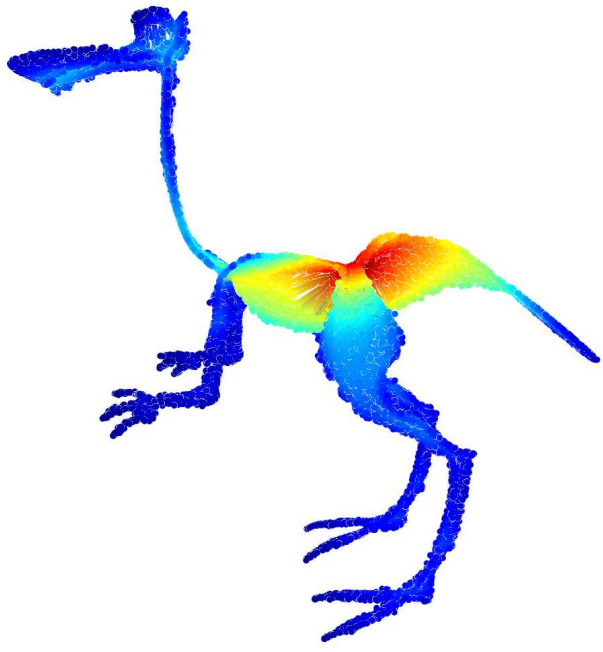

f)

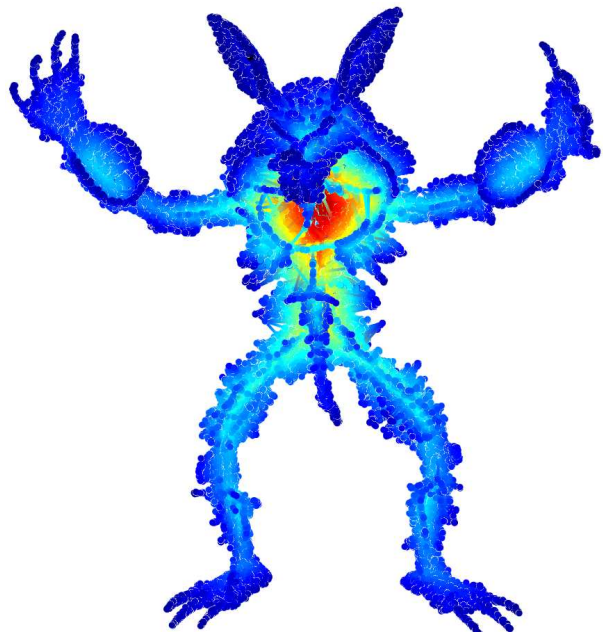

h)

Fig. 8. Qualitative comparisons between the skeletons. The first two rows show the visual effects of our noise removal method with the dinopet model. a), b), c) and d) propose a close view of one foot of this model for the Power Shape and its cleaned version, and for the Scale Axis and its cleaned version. e) is a full view on the Scale Axis skeleton and f) of its cleaned version. A sur-sampled version of Power Shape in $\mathrm{g}$ ) and its cleaned version in $\mathrm{h}$ ). 
will build a multi-resolution shape representation model, allowing processing at different level of detail like rendering, segmenting, or interaction.

\section{References}

1. Amenta, N., Choi, S., Kolluri, R.K.: The power crust. In: 6th ACM symposium on Solid modeling and applications. pp. 249-266. ACM, New York, NY, USA (2001)

2. Amenta, N., Choi, S., Kolluri, R.K.: The power crust, unions of balls, and the medial axis transform. Comput. Geom. Theory Appl. 19(2-3), 127-153 (2001)

3. Aspert, N., Santa-Cruz, D., Ebrahimi, T.: Mesh: Measuring errors between surfaces using the hausdorff distance. In: IEEE International Conference on Multimedia and Expo. vol. I, pp. $705-708$ (2002)

4. Au, O.K.C., Tai, C.L., Chu, H.K., Cohen-Or, D., Lee, T.Y.: Skeleton extraction by mesh contraction. ACM Trans. Graph. 27, 44:1-44:10 (2008)

5. Aylward, S.R., Jomier, J., Weeks, S., Bullitt, E.: Registration and analysis of vascular images. International Journal of Computer Vision 55(2-3), 123-138 (2003)

6. Blum, H.: A Transformation for Extracting New Descriptors of Shape. In: Models for the Perception of Speech and Visual Form, pp. 362-380. MIT Press, Cambridge (1967)

7. Boissonnat, J.D., Oudot, Steve, Y.: Provably good sampling and meshing of surfaces. Graphical Models 67, 405-451 (2005)

8. Brady, M.J., Asada, H.: Smooth local symmetries and their implementations. International Journal of Robotic Research (1984)

9. Brandt, J.: Convergence and continuity criteria for discrete approximations of the continuous planar skeleton. Computer Vision, Graphics, and Image Processing: Image Understanding 59(1), 116 - 124 (1994)

10. Chazal, F., Lieutier, A.: Stability and homotopy of a subset of the medial axis. In: 9th ACM symposium on Solid modeling and applications. pp. 243-248. Eurographics Association, Aire-la-Ville, Switzerland, Switzerland (2004)

11. Cornea, N.D., Silver, D., Min, P.: Curve-skeleton properties, applications, and algorithms. IEEE Trans. on Visualization and Computer Graphics 13, 530-548 (2007)

12. Delamé, T., Roudet, C., Faudot, D.: From a medial surface to a mesh. Computer Graphics Forum 31(5), 1637-1646 (2012)

13. Dey, T.K., Zhao, W.: Approximating the medial axis from the voronoi diagram with a convergence guarantee. Algorithmica 38(1), 179-200 (2003)

14. Giesen, J., Miklos, B., Pauly, M., Wormser, C.: The scale axis transform. In: 25th annual symposium on Computational geometry. pp. 106-115. ACM, New York, NY, USA (2009)

15. Kruithof, N.G.H., Vegter, G.: Meshing skin surfaces with certified topology. In: 9th International Conference on Computer Aided Design and Computer Graphics. pp. 287-294. CAD-CG '05, IEEE Computer Society

16. Leyton, M.: Symmetry-curvature duality. Computer Vision, Graphics, and Image Processing 38, 327-341 (1987)

17. Miklos, B., Giesen, J., Pauly, M.: Discrete scale axis representations for 3d geometry. ACM Trans. Graph. 29 (2010)

18. Tagliasacchi, A., Zhang, H., Cohen-Or, D.: Curve skeleton extraction from incomplete point cloud. ACM Trans. Graph. 28, 71:1-71:9 (2009)

19. Tam, R., Heidrich, W.: Shape simplification based on the medial axis transform. In: 14th IEEE Visualization 2003 (VIS'03). pp. 63-. IEEE Computer Society, Washington, DC, USA (2003) 